\title{
LEFT ABSOLUTELY FLAT GENERALIZED INVERSE SEMIGROUPS
}

\author{
SYDNEY BULMAN - FLEMING ${ }^{1}$ AND KENNETH MCDOWELL ${ }^{2}$
}

\begin{abstract}
A semigroup $S$ is called (left, right) absolutely flat if all of its (left, right) $S$-sets are flat. $S$ is a (left, right) generalized inverse semigroup if $S$ is regulai and its set of idempotents $E(S)$ is a (left, right) normal band (i.e. a strong semilattice of (left zero, right zero) rectangular bands). In this paper it is proved that a generalized inverse semigroup $S$ is left absolutely flat if and only if $S$ is a right generalized inverse semigroup and the (nonidentity) structure maps of $E(S)$ are constant. In particular all inverse semigroups are left (and right) absolutely flat (see [1]). Other consequences are derived.
\end{abstract}

1. Introduction. Let $S$ be a semigroup. $S$-Ens (respectively, Ens- $S$ ) will denote the class of all left (right) $S$-sets. For $A \in$ Ens-S and $B \in S$-Ens, let $\tau$ denote the smallest equivalence relation on $A \times B$ containing all pairs $((a s, b),(a, s b))$ for $a \in A, b \in B$, and $s \in S$. The tensor product $A \otimes B$ (or, more precisely, $A \otimes_{S} B$ ) is defined to be the set $(A \times B) / \tau$, and possesses the customary universal mapping property with respect to balanced maps from $A \times B$ to an arbitrary set. For $a \in A$ and $b \in B, a \otimes b$ represents the $\tau$-class of $(a, b) . B$ is called flat (in $S$-Ens) if and only if, for all embeddings $A \rightarrow C$ in Ens-S, the induced map $A \otimes B \rightarrow C \otimes B$ is an embedding. $S$ is called left absolutely flat if all of its left $S$-sets are flat. Right absolute flatness is defined similarly, and $S$ is called absolutely flat if it is both left and right absolutely flat.

In [4], M. Kil'p proves that every left absolutely flat semigroup is regular, and in [5] that every inverse union of groups is absolutely flat. The present authors show that, in fact, every inverse semigroup is absolutely flat [1] and in the same paper characterize those Rees matrix semigroups (with or without zero) which are left absolutely flat. Furthermore, it is known that every left absolutely flat union of groups must be a semilattice of right groups (see, for example [6]). In this paper we shall characterize the generalized inverse semigroups (and in particular the strong semilattices of right groups) which are left absolutely flat.

In his study of amalgamation of semigroups, T. E. Hall developed the Free Representation Extension Property [3]. A semigroup is left absolutely flat if and only if it has this property in the class of all semigroups (see [2, Proposition 1.1]).

Received by the editors July 26, 1984.

1980 Mathematics Subject Classification. Primary 20M10; Secondary 20M50.

Key words and phrases. Generalized inverse semigroup, absolutely flat semigroup, normal band.

${ }^{1}$ Research supported by Natural Sciences and Engineering Research Council grant A4494.

${ }^{2}$ Research supported by Natural Sciences and Engineering Research Council grant A9241. 
Amalgamation bases (in the class of all semigroups) often have this property and its dual, i.e. they are often absolutely flat. The authors intend to employ the material developed in this paper in subsequent studies on amalgamation.

2. Left absolutely flat generalized inverse semigroups. If $S$ is a semigroup, $S^{1}$ denotes the monoid obtained by adjoining a new identity element 1 to $S$. Let $A \in$ Ens- $S, a, \hat{a} \in A, B \in S$-Ens, and $b, \hat{b} \in B$. Following Lemma 1.2 of [1] it is easy to show that $a \otimes b=\hat{a} \otimes \hat{b}$ in $A \otimes_{S} B$ if and only if there exist $a_{1}, \ldots, a_{n} \in A$, $b_{2}, \ldots, b_{n} \in B, s_{1}, \ldots, s_{n} t_{1}, \ldots, t_{n} \in S^{1}$ such that

$$
\begin{array}{rlrl}
a & =a_{1} s_{1} & \\
a_{1} t_{1} & =a_{2} s_{2} & s_{1} b & =t_{1} b_{2} \\
a_{2} t_{2} & =a_{3} s_{3} & s_{2} b_{2} & =t_{2} b_{3} \\
& \vdots & & \vdots \\
a_{n} t_{n} & =\hat{a} & s_{n} b_{n} & =t_{n} \hat{b}
\end{array}
$$

(where it is assumed that $S^{1}$ acts unitally on $A$ and $B$ ). The system of equalities above is called a scheme over $A$ and $B$ of length $n$ joining $(a, b)$ to $(\hat{a}, \hat{b})$. From this description we see that a left $S$-set $B$ is flat if and only if, for every right $S$-set $A$, and every $a, \hat{a} \in A, b, \hat{b} \in B$ such that there exists a scheme over $A$ and $B$ joining $(a, b)$ to $(\hat{a}, \hat{b})$, there exists a scheme (of possibly different length) over $a S^{1} \cup \hat{a} S^{1}$ and $B$ joining $(a, b)$ to $(\hat{a}, \hat{b})$. (See Lemma 2.2 of [1].)

A regular semigroup $S$ is called a (left, right) generalized inverse semigroup (see [7]) provided its set of idempotents $E(S)$ forms a (left, right) normal band, or, equivalently, provided $S$ is regular and

$$
(x e f=x f e, e f x=f e x) \quad x e f y=x f e y
$$

for all $x, y \in S, e, f \in E(S)$.

Proposition 2.1. Every left absolutely flat generalized inverse semigroup is a right generalized inverse semigroup.

Proof. Let $S$ be a left absolutely flat generalized inverse semigroup. We need only show that if $e, f \in E(S)$, efe $=e$, and $f e f=f$, then $e f=f$. Let $\theta_{L}(e, f)$ denote the smallest left congruence on $S^{1}$ which identifies $e$ and $f$. Clearly $e \otimes \overline{1}=f \otimes \overline{1}$ in $S^{1} \otimes S^{1} / \theta_{L}(e, f)$. Hence $e \otimes \overline{1}=f \otimes \overline{1}$ in $(e S \cup f S) \otimes S^{1} / \theta_{L}(e, f)$ and so either $e=f$ (in which case $e f=f$ ) or there exist $a_{1}, \ldots, a_{n} \in e S \cup f S$ and $s_{1}, \ldots, s_{n} t_{1}, \ldots, t_{n}$ $\in S^{1}$ such that $\left\{s_{i}, t_{i}\right\}=\{e, f\}$ for $i=1, \ldots, n$ and

$$
\begin{aligned}
e & =a_{1} s_{1} \\
a_{1} t_{1} & =a_{2} s_{2} \\
& \vdots \\
a_{n} t_{n} & =f
\end{aligned}
$$

(see [1, Lemma 1.1]). By induction we now establish $e=a_{i} f e$ for $i=1, \ldots, n$. If $i=1, a_{1} f e=a_{1} f s_{1} f e=a_{1} s_{1} f e=e f e=e$. Now suppose $a_{k-1} f e=e$ for some $k$, $1<k<n$. Then $a_{k} f e=a_{k} f s_{k} f e=a_{k} s_{k} f e=a_{k-1} t_{k-1} f e=a_{k-1} f t_{k-1} f e=a_{k-1} f e$ $=e$, and the induction is complete. In particular $e=a_{n} f e$ and so $e f=a_{n} f e f=$ $a_{n} f t_{n} f=a_{n} t_{n} f=f$ as required. 
We shall need the following lemma to establish a second condition which is necessary for a (right) generalized inverse semigroup to be absolutely flat.

LEMMA 2.2. Let $S$ be a right generalized inverse semigroup, and let $e_{1}, e_{2}, f_{1}$, $f_{2} \in E(S)$ be such that $e_{1} \mathscr{R} e_{2}, f_{1} \mathscr{R} f_{2}, f_{1} e_{1}=f_{1}$ and $f_{2} e_{2}=f_{2}$. Then $\left(f_{1}, f_{2}\right) \in$ $\theta_{L}\left(e_{1}, f_{1}\right) \vee \theta_{L}\left(e_{2}, f_{2}\right)$ implies $f_{1}=f_{2}$.

Proof. It will be convenient to introduce the abbreviations $\theta_{i}=\theta_{L}\left(e_{i}, f_{i}\right), i=1,2$, and $\Phi=\theta_{1} \circ \theta_{2}$. Since $\theta_{1} \vee \theta_{2}=\bigcup_{n=1}^{\infty} \Phi^{n}$, it suffices to prove by induction that, for every $n \in N,\left(f_{1}, f_{2}\right) \in \Phi^{n}$ implies $f_{1}=f_{2}$. In the process we shall use the (easily verified) fact that, for $x, y \in S^{1},(x, y) \in \theta_{i}$ if and only if $(x=y)$ or $\left(x e_{i}=x, y e_{i}=\right.$ $y$, and $\left.x f_{i}=y f_{i}\right), i=1,2$.

First, then, suppose $\left(f_{1}, f_{2}\right) \in \Phi^{1}$, so that $f_{1} \theta_{1} z \theta_{2} f_{2}$ for some $z \in S^{1}$. If $z=f_{1}$ then $\left(f_{1}, f_{2}\right) \in \theta_{2}$ results, which in particular entails $f_{1} e_{2}=f_{1}$. But also $f_{1} e_{2}=f_{2} e_{2}=f_{2}$, so $f_{1}=f_{2}$ as claimed. A similar argument succeeds if $z=f_{2}$. If $f_{1} \neq z \neq f_{2}$ then we have $f_{1} e_{1}=f_{1}, z e_{1}=z, f_{1}=z f_{1}$ (since $\left.\left(f_{1}, z\right) \in \theta_{1}\right)$ and $z e_{2}=z, f_{2} e_{2}=f_{2}, z f_{2}=f_{2}$ (since $\left.\left(z, f_{2}\right) \in \theta_{2}\right)$. Note that $z \neq 1$. If $z^{\prime}$ denotes any inverse of $z$ in $S$, and we recall that $z z^{\prime}, z^{\prime} z \in E(S)$, then we may calculate $f_{1}=z f_{1}=z f_{1} e_{1}=z f_{1} z^{\prime} z e_{1}=$ $z f_{1} z^{\prime} z=z f_{1} z^{\prime} z e_{2}=z f_{1} e_{2}=z f_{2}=f_{2}$, as desired.

Now assume that $\left(f_{1}, f_{2}\right) \in \Phi^{n}$ implies $f_{1}=f_{2}$ for some $n \geqslant 1$, and let $z_{1}, z_{2} \in S^{1}$ be such that $f_{1} \Phi^{n} z_{1} \theta_{1} z_{2} \theta_{2} f_{2}$. If $z_{1}=z_{2}$ then $\left(f_{1}, f_{2}\right) \in \Phi^{n}$ immediately results (since $\Phi^{n} \circ \theta_{2}=\Phi^{n}$ ) and the inductive hypothesis finishes the argument. Otherwise, if $z_{1} \neq z_{2}$ (and without loss of generality we assume $z_{i} \neq f_{i}, i=1,2$ ) we see that $f_{1}=f_{2} f_{1}=z_{2} f_{2} f_{1}=z_{2} f_{1}$, which together with $f_{1} e_{1}=f_{1}$ and $z_{2} e_{1}=z_{2}$ implies $\left(f_{1}, z_{2}\right) \in \theta_{1}$. Hence, $f_{1} \Phi f_{2}$ which by the $n=1$ case yields $f_{1}=f_{2}$ and the proof is complete.

Every right normal band $E$ is a strong semilattice of right zero bands, i.e. $E=\mathscr{S}\left(\Gamma ; R_{\gamma} ; \phi_{\alpha, \beta}\right)$ where $\Gamma$ is a semilattice, each $R_{\gamma}(\gamma \in \Gamma)$ is a right zero band, $E=\cup_{\gamma \in \Gamma} R_{\gamma}$, and the maps $\phi_{\alpha, \beta}: R_{\alpha} \rightarrow R_{\beta}(\alpha \geqslant \beta)$ are the structure maps. We shall say $E$ has constant structure maps if $\phi_{\alpha, \beta}$ is a constant function whenever $\alpha>\beta$ $(\alpha, \beta \in \Gamma)$. It is not difficult to prove that $E$ has constant structure maps if and only if

$$
(\forall e, f, g \in E)(e f g=f g \text { or } e f g=e g f) \text {. }
$$

Proposition 2.3. If $S$ is a left absolutely flat right generalized inverse semigroup then $E(S)$ has constant structure maps.

Proof. Refer to (2) and assume $e, f, g \in E(S)$ and $e f g \neq f g$. Let $e_{1}=f g, e_{2}=g f$, $f_{1}=e f g$ and $f_{2}=e g f$ and notice that $e_{1}, e_{2}, f_{1}, f_{2}$ satisfy the hypotheses of Lemma 2.2. As before, suppose $\theta_{i}=\theta_{L}\left(e_{i}, f_{i}\right), i=1,2$, and let $\theta_{R}\left(e_{1}, e_{2}\right)$ denote the smallest right congruence on $S$ which identifies $e_{1}$ and $e_{2}$. Note that the only nonsingleton class of $\theta_{R}\left(e_{1}, e_{2}\right)$ is $\left\{e_{1}, e_{2}\right\}$. Because of this and the fact that $e f g \neq f g, \mu$ : $f_{1} S \cup f_{2} S \rightarrow S / \theta_{R}\left(e_{1}, e_{2}\right)$ defined by $\mu(s)=\bar{s}$ for all $s \in f_{1} S \cup f_{2} S$ is a monomorphism. Hence,

$$
\mu \otimes \mathrm{id}:\left(f_{1} S \cup f_{2} S\right) \otimes S^{1} / \theta_{1} \vee \theta_{2} \rightarrow S / \theta_{R}\left(e_{1}, e_{2}\right) \otimes S^{1} / \theta_{1} \vee \theta_{2}
$$


is injective. Since $\bar{f}_{1} \otimes \overline{1}=\bar{f}_{2} \otimes \overline{1}$ in $S / \theta_{R}\left(e_{1}, e_{2}\right) \otimes S^{1} / \theta_{1} \vee \theta_{2}$, it follows that $f_{1} \otimes \overline{1}=f_{2} \otimes \overline{1}$ in $\left(f_{1} S \cup f_{2} S\right) \otimes S^{1} / \theta_{1} \vee \theta_{2}$. We therefore obtain a scheme

$$
\begin{array}{rlrl}
f_{1} & =a_{1} s_{1} & & \\
a_{1} t_{1} & =a_{2} s_{2} & s_{1} \overline{1} & =t_{1} \bar{b}_{2} \\
\vdots & & \vdots \\
a_{n} t_{n} & =f_{2} & s_{n} \bar{b}_{n}=t_{n} \overline{1}
\end{array}
$$

where for $i=1, \ldots, n, a_{i} \in f_{1} S \cup f_{2} S, s_{i}, t_{i} \in S^{1}$ and $b_{2}, \ldots, b_{n} \in S^{1}$. From this it follows that $\left(f_{1}, f_{2}\right) \in \theta_{1} \vee \theta_{2}$ which by Lemma 2.2 yields $f_{1}=f_{2}$, i.e. efg $=e g f$, as required.

Our main result is the following theorem in which we demonstrate that the necessary conditions developed in Propositions 2.1 and 2.3 are also sufficient.

THEOREM 2.4. Let $S$ be a right generalized inverse semigroup in which $E(S)$ has constant structure maps. Then $S$ is left absolutely flat.

Proof. Suppose $A \in$ Ens- $S$ and $B \in S$-Ens. We will prove by induction on $n$ that any scheme

$$
\begin{array}{rlrl}
a & =a_{1} s_{1} & \\
a_{1} t_{1} & =a_{2} s_{2} & & s_{1} b=t_{1} b_{2} \\
a_{2} t_{2} & =a_{3} s_{3} & s_{2} b_{2}=t_{2} b_{3} \\
& \vdots & & \vdots \\
a_{n} t_{n} & =\hat{a} & s_{n} b_{n}=t_{n} \hat{b}
\end{array}
$$

over $A$ and $B$ joining $(a, b)$ to $(\hat{a}, \hat{b})$ may be replaced by one over $a S^{1} \cup \hat{a} S^{1}$ and $B$. Throughout this proof $s_{i}^{\prime}\left(\right.$ resp. $\left.t_{i}^{\prime}\right), i=1, \ldots, n$, will denote a fixed inverse of $s_{i}\left(t_{i}\right)$ in $S$.

If $n=1(\Sigma)$ is

$$
\begin{aligned}
& a=a_{1} s_{1} \\
& a_{1} t_{1}=\hat{a} \quad s_{1} b=t_{1} \hat{b} .
\end{aligned}
$$

If $s_{1}=1$ or $t_{1}=1$ the scheme itself serves as the replacement. Otherwise the following scheme serves:

$$
\begin{array}{rlrl}
a & =\left(a s_{1}^{\prime}\right) s_{1} & & \\
\left(a s_{1}^{\prime}\right) t_{1} & =\left(a s_{1}^{\prime} t_{1} t_{1}^{\prime}\right) t_{1} & & s_{1} b=t_{1} \hat{b} \\
\left(a s_{1}^{\prime} t_{1} t_{1}^{\prime}\right) s_{1} & =\left(\hat{a} t_{1}^{\prime}\right) s_{1} & t_{1} \hat{b}=s_{1} b \\
\left(\hat{a} t_{1}^{\prime}\right) t_{1} & =\hat{a} & s_{1} b & =t_{1} \hat{b}
\end{array}
$$

Only the third line on the left may need explanation:

$$
\begin{aligned}
a s_{1}^{\prime} t_{1} t_{1}^{\prime} s_{1} & =a_{1} s_{1} s_{1}^{\prime} t_{1} t_{1}^{\prime} s_{1} \\
& =a_{1} t_{1} t_{1}^{\prime} s_{1} s_{1}^{\prime} s_{1} \quad(\text { by (1)) } \\
& =\hat{a} t_{1}^{\prime} s_{1} .
\end{aligned}
$$


Now assume that for some $n>1$ all schemes of length less than $n$ can be "replaced" and consider $(\Sigma)$ as above. Before proceeding we first note that whenever $a_{i} t_{i}=a_{i+1} s_{i+1} \in a S^{1}$ for some $i=1, \ldots, n-1$, then $(\Sigma)$ can be resolved into the two shorter schemes

$$
\begin{array}{rlrl}
a & =a_{1} s_{1} & \\
a_{1} t_{1} & =a_{2} s_{2} & s_{1} b & =t_{1} b_{2} \\
& \vdots & & \vdots \\
a_{i} t_{i} & =a_{i+1} s_{i+1} & s_{i} b_{i}=t_{i} b_{i+1}
\end{array}
$$

and

$$
\begin{array}{rlrl}
a_{i} t_{i} & =a_{i+1} s_{i+1} & \\
a_{i+1} t_{i+1} & =a_{i+2} s_{i+2} & s_{i+1} b_{i+1} & =t_{i+1} b_{i+2} \\
& \vdots & \vdots \\
a_{n} t_{n} & =\hat{a} & s_{n} b_{n} & =t_{n} \hat{b} .
\end{array}
$$

By the inductive hypothesis, the first scheme may be replaced by one over $a S^{1}$ and $B$ joining $(a, b)$ to $\left(a_{i+1} s_{i+1}, b_{i+1}\right)$ and the second may be replaced by one over $a S^{1} \cup \hat{a} S^{1}$ and $B$ joining $\left(a_{i} t_{i}, b_{i+1}\right)$ to $(\hat{a}, \hat{b})$. The two new schemes may then be spliced together to join $(a, b)$ and $(\hat{a}, \hat{b})$ over $a S^{1} \cup \hat{a} S^{1}$ and $B$ as required.

Now we show that without loss of generality we may assume $s_{i} \neq 1$ and $t_{i} \neq 1$ for all $i$. In fact, if $s_{1}=1$ then $a_{1} t_{1} \in a S^{1}$ and, hence, the scheme may be replaced using the preceding argument. If $s_{i}=1$ for some $i>1$ it is an easy matter to see that $(\Sigma)$ may be replaced by a scheme of length $n-1$ allowing the use of the inductive hypothesis. If $t_{i}=1$ for some $i$, similar considerations apply.

We henceforth assume that $s_{i}, t_{i} \in S$ for $i=1, \ldots, n$. It will be useful to establish the following notation:

$$
\begin{array}{ll}
z_{1}=s_{1}^{\prime}, & z_{i+1}=z_{i} t_{i} s_{i+1}^{\prime}, \\
z_{1}^{\prime}=s_{1}, & z_{i+1}^{\prime}=s_{i+1} t_{i}^{\prime} z_{i}^{\prime}, \\
w_{n}=t_{n}^{\prime}, & w_{i}=w_{i+1} s_{i+1} t_{i}^{\prime}, \\
w_{n}^{\prime}=t_{n}, & w_{i}^{\prime}=t_{i} s_{i+1}^{\prime} w_{i+1}^{\prime}
\end{array}
$$

for $1 \leqslant i \leqslant n-1$. The following equalities can be verified for $1 \leqslant i \leqslant n$ :

$$
\begin{gathered}
w_{1} s_{1}=w_{i} z_{i}^{\prime}, \\
z_{n} t_{n}=z_{i} w_{i}^{\prime}, \\
a z_{i}=a_{i} z_{i}^{\prime} z_{i}, \\
\hat{a} w_{i}=a_{i} w_{i}^{\prime} w_{i} .
\end{gathered}
$$

For example, to establish (7), we use induction on $i$. If $i=1$ the result is clear. 
Otherwise,

$$
\begin{aligned}
a z_{i+1} & =a z_{i} t_{i} s_{i+1}^{\prime} & & \text { (by (3)) } \\
& =a_{i} z_{i}^{\prime} z_{i} t_{i} s_{i+1}^{\prime} & & \text { (inductive hypothesis) } \\
& =a_{i} z_{i}^{\prime} z_{i} t_{i} t_{i}^{\prime} t_{i} s_{i+1}^{\prime} & & \\
& =a_{i} t_{i} t_{i}^{\prime} z_{i}^{\prime} z_{i} t_{i} s_{i+1}^{\prime} & & (\text { by (1)) } \\
& =a_{i+1} s_{i+1} t_{i}^{\prime} z_{i}^{\prime} z_{i} t_{i} s_{i+1}^{\prime} & & \\
& =a_{i+1} z_{i+1}^{\prime} z_{i+1} & & (\text { by (3) and (3')). }
\end{aligned}
$$

Now let

$$
e_{i}=t_{i}^{\prime} z_{i}^{\prime} z_{i} t_{i}
$$

and

$$
f_{i}=s_{i}^{\prime} w_{i}^{\prime} w_{i} s_{i}
$$

for $i=1, \ldots, n$. If $e_{i} t_{i}^{\prime} t_{i} s_{i+1}^{\prime} s_{i+1}=t_{i}^{\prime} t_{i} s_{i+1}^{\prime} s_{i+1}$ for some $i(1 \leqslant i \leqslant n-1)$, then $t_{i} e_{i} s_{i+1}^{\prime} s_{i+1}=t_{i} s_{i+1}^{\prime} s_{i+1}$ and so

$$
\begin{array}{rlrl}
a_{i} t_{i} & =a_{i} t_{i} s_{i+1}^{\prime} s_{i+1} & \\
& =a_{i} t_{i} e_{i} s_{i+1}^{\prime} s_{i+1} & \\
& =a_{i} t_{i} t_{i}^{\prime} z_{i}^{\prime} z_{i} t_{i} s_{i+1}^{\prime} s_{i+1} & & (\text { by (9)) } \\
& =a_{i} z_{i}^{\prime} z_{i} t_{i} s_{i+1}^{\prime} s_{i+1} & & (\text { by (1)) } \\
& =a z_{i} t_{i} s_{i+1}^{\prime} s_{i+1} & & (\text { by (7)) }
\end{array}
$$

which shows $a_{i} t_{i} \in a S^{1}$ and a previous argument applies. If $f_{i+1} s_{i+1}^{\prime} s_{i+1} t_{i}^{\prime} t_{i}=$ $s_{i+1}^{\prime} s_{i+1} t_{i}^{\prime} t_{i}$ for some $i(1 \leqslant i \leqslant n-1)$ the proof is similar.

Finally, by (2), we may assume

$$
e_{i} t_{i}^{\prime} t_{i} s_{i+1}^{\prime} s_{i+1}=e_{i} s_{i+1}^{\prime} s_{i+1} t_{i}^{\prime} t_{i}
$$

and

$$
f_{i+1} t_{i}^{\prime} t_{i} s_{i+1}^{\prime} s_{i+1}=f_{i+1} s_{i+1}^{\prime} s_{i+1} t_{i}^{\prime} t_{i}
$$

for all $i(1 \leqslant i \leqslant n-1)$. (11) and (12) imply

$$
s_{i+1} e_{i}=z_{i+1}^{\prime} z_{i+1} s_{i+1}
$$

and

$$
t_{i} f_{i+1}=w_{i}^{\prime} w_{i} t_{i}
$$

For example,

$$
\begin{aligned}
t_{i} f_{i+1} & =t_{i} t_{i}^{\prime} t_{i} f_{i+1} s_{i+1}^{\prime} s_{i+1} & & \\
& =t_{i} f_{i+1} t_{i}^{\prime} t_{i} s_{i+1}^{\prime} s_{i+1} & & (\text { by }(1)) \\
& =t_{i} f_{i+1} s_{i+1}^{\prime} s_{i+1} t_{i}^{\prime} t_{i} & & (\text { by }(12)) \\
& =t_{i} f_{i+1} t_{i}^{\prime} t_{i} & & \\
& =t_{i} s_{i+1}^{\prime} w_{i+1}^{\prime} w_{i+1} s_{i+1} t_{i}^{\prime} t_{i} & & (\text { by }(10)) \\
& =w_{i}^{\prime} w_{i} t_{i} & & \left(\text { by (4) and }\left(4^{\prime}\right)\right) .
\end{aligned}
$$


Without loss of generality we may assume that $n$, the length of $(\Sigma)$, is even. (Otherwise one could consider the scheme

$$
\begin{array}{rlrl}
a & =\left(a s_{1}^{\prime}\right) s_{1} & & \\
\left(a s_{1}^{\prime}\right) s_{1} & =a_{1} s_{1} & s_{1} b & =s_{1} b \\
a_{1} t_{1} & =a_{2} s_{2} & s_{1} b & =t_{1} b_{2} \\
& \vdots & \vdots \\
a_{n} t_{n} & =\hat{a} & s_{n} b_{n}=t_{n} \hat{b}
\end{array}
$$

of length $n+1$ joining $(a, b)$ to $(\hat{a}, \hat{b})$, which still possesses properties $(11)$ and (12).) We now show that $(\Sigma)$ may be replaced by the scheme

$$
\begin{aligned}
& a=\left(a z_{1}\right) s_{1} \\
& \left(a z_{1}\right) t_{1}=\left(a z_{2}\right) s_{2} \quad s_{1} b=t_{1} b_{2} \\
& \left(a z_{n-1}\right) t_{n-1}=\left(a z_{n}\right) s_{n} \\
& s_{n-1} b_{n-1}=t_{n-1} b_{n} \\
& \left(a z_{n}\right) t_{n}=\left(a z_{n} t_{n} w_{n}\right) t_{n} \\
& \left(a z_{n} t_{n} w_{n}\right) s_{m}=\left(a z_{n} t_{n} w_{n-1}\right) t_{n-1} \\
& s_{n} b_{n}=t_{n} \hat{b} \\
& t_{n} \hat{b}=s_{n} b_{n} \\
& \left(a z_{n} t_{n} w_{n / 2+2}\right) s_{n / 2+2}=\left(a z_{n} t_{n} w_{n / 2+1}\right) t_{n / 2+1} \\
& t_{n / 2+2} b_{n / 2+3}=s_{n / 2+2} b_{n / 2+2} \\
& \left(a z_{n} t_{n} w_{n / 2+1}\right) s_{n / 2+1}=\left(\hat{a} w_{1} s_{1} z_{n / 2}\right) t_{n / 2} \\
& t_{n / 2+1} b_{n / 2+2}=s_{n / 2+1} b_{n / 2+1} \\
& \left(\hat{a} w_{1} s_{1} z_{n / 2}\right) s_{n / 2}=\left(\hat{a} w_{1} s_{1} z_{n / 2-1}\right) t_{n / 2-1} \\
& t_{n / 2} b_{n / 2+1}=s_{n / 2} b_{n / 2} \\
& \left(\hat{a} w_{1} s_{1} z_{2}\right) s_{2}=\left(\hat{a} w_{1} s_{1} z_{1}\right) t_{1} \\
& \left(\hat{a} w_{1} s_{1} z_{1}\right) s_{1}=\left(\hat{a} w_{1}\right) s_{1} \\
& t_{2} b_{3}=s_{2} b_{2} \\
& t_{1} b_{2}=s_{1} b \\
& \left(\hat{a} w_{1}\right) t_{1}=\left(\hat{a} w_{2}\right) s_{2} \\
& s_{1} b=t_{1} b_{2} \\
& \left(\hat{a} w_{n-1}\right) t_{n-1}=\left(\hat{a} w_{n}\right) s_{n} \\
& \left(\hat{a} w_{n}\right) t_{n}=\hat{a} \\
& \begin{aligned}
s_{n-1} b_{n-1} & =t_{n-1} b_{n} \\
s_{n} b_{n} & =t_{n} \hat{b} .
\end{aligned}
\end{aligned}
$$

We must check that these equalities hold. Because the equalities on the right appear in the original scheme we need only consider those on the left. The reader will notice that these have been presented in five groups.

That the first equation in the first group holds is obvious. Moreover, for $1 \leqslant i \leqslant n-1$,

$$
\begin{aligned}
a z_{i} t_{i} & =a_{i} z_{i}^{\prime} z_{i} t_{i} \\
& =a_{i} t_{i} t_{i}^{\prime} z_{i}^{\prime} z_{i} t_{i} \\
& =a_{i+1} s_{i+1} t_{i}^{\prime} z_{i}^{\prime} z_{i} t_{i} \\
& =a_{i+1} s_{i+1} e_{i} \\
& =a_{i+1} z_{i+1}^{\prime} z_{i+1} s_{i+1} \\
& =a z_{i+1} s_{i+1}
\end{aligned}
$$

The equations in the fifth group hold for reasons of symmetry. 
That the first equation in the second group holds is clear. Furthermore, for $n / 2+1 \leqslant i \leqslant n-1$,

$$
\begin{aligned}
a z_{n} t_{n} w_{i+1} s_{i+1} & =a z_{i+1} w_{i+1}^{\prime} w_{i+1} s_{i+1} & & (\text { by }(6)) \\
& =a z_{i} t_{i} s_{i+1}^{\prime} w_{i+1}^{\prime} w_{i+1} s_{i+1} & & (\text { by }(3)) \\
& =a z_{i} t_{i} f_{i+1} & & (\text { by }(10)) \\
& =a z_{i} w_{i}^{\prime} w_{i} t_{i} & & (\text { by (14)) } \\
& =a z_{n} t_{n} w_{i} t_{i} & & (\text { by }(6)) .
\end{aligned}
$$

The equations in the fourth group also hold, for analogous reasons. Finally, the middle equation holds. In fact,

$$
\begin{aligned}
a z_{n} t_{n} w_{n / 2+1} s_{n / 2+1} & =a z_{n / 2} w_{n / 2}^{\prime} w_{n / 2+1} s_{n / 2+1} \\
& =a_{n / 2} z_{n / 2}^{\prime} z_{n / 2} w_{n / 2}^{\prime} w_{n / 2+1} s_{n / 2+1} \\
& =a_{n / 2} z_{n / 2}^{\prime} z_{n / 2} t_{n / 2} s_{n / 2+1}^{\prime} w_{n / 2+1}^{\prime} w_{n / 2+1} s_{n / 2+1} \\
& =a_{n / 2} z_{n / 2}^{\prime} z_{n / 2} t_{n / 2} f_{n / 2+1} \\
& =a_{n / 2} z_{n / 2}^{\prime} z_{n / 2} w_{n / 2}^{\prime} w_{n / 2} t_{n / 2} \\
& =a_{n / 2} w_{n / 2}^{\prime} w_{n / 2} z_{n / 2}^{\prime} z_{n / 2} t_{n / 2} \\
& =\hat{a} w_{n / 2} z_{n / 2}^{\prime} z_{n / 2} t_{n / 2} \\
& =\hat{a} w_{1} s_{1} z_{n / 2} t_{n / 2}
\end{aligned}
$$

This completes the proof of Theorem 2.4.

Among the consequences of Theorem 2.4 are

COROllary 2.5 [1, TheOREM 4.2]. Inverse semigroups are absolutely flat.

COROLlary 2.6. Let $S$ be a strong semilattice of completely simple semigroups. Then $S$ is left absolutely flat if and only if $S$ is a strong semilattice of right groups and $E(S)$ has constant structure maps.

Proof. If $S$ is left absolutely flat, then each of its completely simple components must be a right group [6, or $\mathbf{1}$, proof of Theorem 4.3]. Hence $E(S)$ is right normal and has constant structure maps by Theorem 2.4 . The converse clearly holds.

Corollary 2.7. (1) A normal band is left absolutely flat if and only if it is right normal and has constant structure maps.

(2) $A$ right normal band is left absolutely flat if and only if it has constant structure maps.

(3) A left normal band is left absolutely flat if and only if it is a semilattice.

\section{REFERENCES}

1. S. D. Bulman-Fleming and K. McDowell, Absolutely flat semigroups, Pacific J. Math. 107 (1983), $319-333$

2. F___ Flatness and amalgamation in semigroups, Semigroup Forum 29 (1984), 337-342.

3. T. E. Hall, Representation extension and amalgamation for semigroups, Quart. J. Math. Oxford (2) 29 (1978), 309-334. 
4. M. Kil'p, On flat polygons, Uchen. Zap. Tartu Un-ta 253 (1970), 66-72.

5. ___ On homological classification of monoids, Siberian Math. J. 13 (1972), 396-401.

6. Left completely flat monoids that are unions of groups (Russian. Estonian and English summaries), Tartu Riikl. Ul. Toimetised No. 556, Trudy Mat. Meh. (1981), 33-37.

7. M. Yamada, Regular semigroups whose idempotents satisfy permutation identities, Pacific J. Math. 21 (1967), 371-392.

Department of Mathematics, Wilfrid laurier University, Waterloo, Ontario, Canada N2L $3 \mathrm{C} 5$ 